\title{
Halitosis and tongue coating in patients with erosive gastroesophageal reflux disease versus nonerosive gastroesophageal reflux disease
}

\author{
Karin Kislig • Clive H. Wilder-Smith • \\ Michael M. Bornstein • Adrian Lussi • Rainer Seemann
}

Received: 22 September 2011 / Accepted: 22 February 2012 /Published online: 23 March 2012

(C) Springer-Verlag 2012

\begin{abstract}
Objective The aim of this study was to investigate whether patients with diagnosed erosive gastroesophageal reflux disease (ERD) have an increased probability of halitosis and tongue coating compared to patients with nonerosive gastroesophageal reflux disease (NERD).

Materials and methods Sixty-six patients (33 males and 33 females) were recruited for the study and received an upper gastrointestinal endoscopy. The presence of ERD $(n=31)$ and NERD $(n=35)$ was classified based on the Los Angeles classification for erosive changes in the esophagus. Additionally, the patients filled in a questionnaire regarding their subjective assessment of halitosis, and an organoleptic assessment of halitosis, a measurement of oral volatile sulfur compounds (VSC) with the Halimeter, and a tongue coating index were performed. ERD and NERD subjects were compared with regard to Halitosis-related clinical and anamnestic findings.

Results No statistically significant difference could be found between ERD and NERD patients regarding tongue coating
\end{abstract}

K. Kislig $(\bowtie) \cdot$ A. Lussi $\cdot$ R. Seemann

Department of Preventive, Restorative and Pediatric Dentistry,

School of Dental Medicine, University of Bern,

Freiburgstrasse 7,

CH-3010 Bern, Switzerland

e-mail: karin.kislig@zmk.unibe.ch

C. H. Wilder-Smith

Gastroenterology Group Practice,

Bern, Switzerland

M. M. Bornstein

Department of Oral Surgery and Stomatology,

School of Dental Medicine, University of Bern,

Bern, Switzerland index, organoleptic scores, and VSC values as well as selfperceived bad taste, tongue coating, and bad breath.

Conclusions These data suggest that halitosis is not typically associated with erosive gastroesophageal reflux disease and the presence of esophageal mucosal damage (ERD patients).

Clinical relevance The data of this investigation support the findings of interdisciplinary bad breath clinics that gastroesophageal reflux disease is not a leading cause for halitosis.

Keywords Halitosis · Reflux disease · ERD · NERD . GERD . Tongue coating

\section{Introduction}

Patients suffering from halitosis exhale a noticeably unpleasant odor in their breath. Halitosis has a high prevalence rate of between 6 and 34\%, depending on the study population [1-4]. Generally, there are two origins of halitosis: oral and extraoral. In the majority of cases (80-90 $\%$ ), halitosis is of oral origin, mostly caused by anaerobic bacteria on the dorsal surface of the tongue [5-7]. These anaerobic bacteria degrade sulfur-containing amino acids to so-called volatile sulfur compounds (VSC). According to data obtained in interdisciplinary bad breath clinics, an oral cause was found in the majority of the cases. Tongue coating $(43.4 \%)$ was the most frequent associated factor, followed by a combination of tongue coating, periodontitis, and gingivitis (18.2\%). In a few cases, periodontitis (7.4\%) and gingivitis $(3.8 \%)$ were determined as the sole underlying factors. Xerostomia was the most important other oral cause $(2.5 \%)$ [8]. 
In a minority of cases, however, halitosis may also be of extraoral origin. Extraoral origins for halitosis are, amongst others, the respiratory tract, pharyngo-tonsilar diseases, or the gastrointestinal tract $[9,10]$. In the last few years, gastrointestinal conditions, especially gastroesophageal reflux diseases (GERD), have been increasingly considered as a possible extraoral source of halitosis [11-16]. Two studies from Korea discovered that VSCs were closely associated with erosive changes of the upper gastrointestinal tract and could be the product of severely inflamed or eroded mucosa. They found a significant difference in VSC levels between patients with erosive gastrointestinal reflux disease (ERD) and nonerosive gastrointestinal reflux disease (NERD) but did not consider oral factors for halitosis such as tongue coating [14, 15]. It has been speculated that gastric pathologies are not the direct source of halitosis but may indirectly lead to ecological changes in the mouth. This change may cause tongue coating and therefore contribute to the most prevalent source for halitosis.

The aim of the present study was to assess subjective and objective parameters for halitosis including tongue coating in patients with ERD and patients with NERD diagnosed by esophago-duodeno-gastroscopy. Our working hypothesis was that patients with diagnosed ERD show elevated halitosis-related parameters compared to patients with NERD.

\section{Material and methods}

\section{Patients}

A total of 66 successive female and male patients were recruited in the period from June 2010 to February 2011. All patients were referred to the Gastroenterology Group Practice in Bern by their general practitioners for evaluation of upper gastrointestinal and reflux symptoms. For inclusion, all participants had to report typical reflux symptoms including heartburn, regurgitation, and dysphagia with (ERD) or without clinical signs of erosive reflux disease (NERD) as detected by esophago-gastro-duodenoscopy. All patients gave their written informed consent to participate in the study, and no patient refused participation. Patients taking antipsychotic drugs, proton-pump inhibitors, or antibiotics within the last 2 weeks or with systemic illness judged relevant by the investigators, including diabetes mellitus, liver diseases, and renal disease were excluded from the present study. Prior to commencement, the study was approved by the standing Ethics Committee of the State of Bern, Switzerland (approval number 198/05).

\section{GERD questionnaire}

The Reflux Disease Questionnaire, validated in German, was used for the assessment of gastrointestinal and specifically
GERD symptoms before endoscopy and further investigation [16]. This questionnaire assesses characteristic GERD symptoms, including retrosternal pain, epigastric pain, heartburn, dysphagia, vomiting, and sour taste in the mouth.

\section{Esophago-duodeno-gastroscopy}

Upper gastrointestinal endoscopy was performed in a standardized fashion by three experienced consultant gastroenterologists with over 15 years of endoscopic experience using Fujinon 450HR (Fujifilm, Tokyo, Japan) endoscopes. Patients arrived after an overnight fast and received standardized sedation using propofol (AstraZeneca, Baar, Switzerland), which was administered by an anesthesiologist. The mucosa was carefully inspected for signs of GERD, and all predisposing factors, such as hiatal hernias, were recorded. The Los Angeles (LA) classification was used for grading of the erosive changes in the esophagus [17]. Grade $A$ is defined as a mucosal break $\leq 5 \mathrm{~mm}$ in length whereas Grade B is $>5 \mathrm{~mm}$. If the mucosal break is continuous between more than two mucosal folds, the score is $\mathrm{C}$, and a mucosal break $\geq 75 \%$ of esophageal circumference is defined as score D. According to these criteria, the patients were classified as ERD or, in case of absence of erosions, as NERD.

\section{Halitosis questionnaire}

After completion of the upper gastrointestinal endoscopy and full recovery from sedation, a second questionnaire including a total of 29 questions focusing on subjective halitosis parameters was completed:

- Medical history regarding known blood, heart, lung, intestinal or renal diseases, and diabetes

- Medications and consumption of alcoholic beverages: never/rarely/daily/several times per day

- Smoking: number of cigarettes smoked per day

- Dental and oral hygiene: How many times per day do you brush your teeth? Do you floss? Do you clean your tongue? Do you use a mouth rinse?

- Halitosis: How often do you suffer from halitosis? Never/ rarely/sometimes/often

- How did you discover you suffer from halitosis? Informed by others/people's behavior towards me/I just know

- Did you ask your dentist for help?

\section{Clinical parameters}

Clinical examinations of the study subjects were always performed between 08:00 and 12:00 h. Participants had fasted for the last $12 \mathrm{~h}$ before the gastroscopy. The clinical 
examination of each study participant began with the organoleptic assessment of halitosis, performed by one experienced dental clinician (K.K.) in a standardized manner using two different indices. The first was the index described by Rosenberg and coworkers [18].

- Grade $0=$ no appreciable odor

- Grade 1 = barely noticeable odor

- Grade 2 = slight but clearly noticeable odor

- Grade $3=$ moderate odor

- Grade 4 = strong odor

- Grade 5 = extremely foul odor

The second index was a simplified index described by Seemann [19].

- Grade $0=$ halitosis not detected

- Grade $1=$ halitosis only diagnosed when the subject was breathing through an open mouth and the observer approached to a distance of about $10 \mathrm{~cm}$

- Grade 2 = halitosis only detected at a distance of about $30 \mathrm{~cm}$ from the subject's mouth

- Grade 3 = halitosis already diagnosed on welcoming the subject, with a distance of approximately $1 \mathrm{~m}$ between the examiner's nose and the subject's mouth

The organoleptic assessment was succeeded by grading of the tongue coating using the following modified grading scale $[20]$.

- Grade $0=$ no tongue coating present

- Grade 1 = light coating of the tongue present (about $10 \%$ of the surface)

- Grade $2=$ moderate coating of the tongue present (10$50 \%$ of the surface)

- Grade $3=$ severe coating of the tongue present $(>50 \%$ of the surface)

In addition to organoleptic measurements, halitosis was also assessed using a commercially available VSC monitor (Halimeter; Interscan, Chatsworth, CA, USA). The monitor was calibrated to zero on ambient air prior to each measurement. Patients were asked to breathe through the nose, with the mouth closed, for $1 \mathrm{~min}$. Then, a straw attached to the monitor was placed at the dorsal posterior part of the tongue, and air was aspirated for analysis. The peak VSC value was recorded in parts per billion (ppb). Three consecutive independent measurements were taken, and the mean value was calculated and used for further analysis.

Statistical analysis

To assess the influence of ERD and NERD on halitosisrelated clinical findings, logistic regression models as well as Kendall's tau correlation coefficients were calculated. The significance level was set to 0.05 . All statistical analysis was performed with the $\mathrm{R} 2.13 .0$ program (http://www.rproject.org/).

\section{Results}

Study population

A total of 66 successive patients, 33 men and 33 women, with a mean age of 53 years (range 18-86) presenting with typical gastroesophageal reflux symptoms were consecutively recruited for the study.

Questionnaire analysis

Most of the participants of the study reported brushing their teeth twice per day $(62.1 \%, 41$ subjects), while $16(24.2 \%)$ subjects indicated brushing three times daily, and 9 study subjects $(20.6 \%)$ reported brushing once per day only. Additionally, 15 subjects reported flossing every day (22.7\%), and 31 were using a mouth rinse (47\%). About one-third of the study population cleaned their tongue $(37.9 \%, 25$ subjects), but only $14(21.2 \%)$ cleaned their tongue on a daily basis.

Twelve participants $(18.2 \%)$ estimated that halitosis was "often" present, 13 (19.7\%) noted "sometimes," and 14 (21.2 \%) "rarely." The majority did not report to suffer from halitosis $(40.9 \%, 27$ subjects). Of the persons with halitosis $(59 \%$, 39 subjects), 15 had been informed by other that they had oral malodor (38.5\%). Only five out of the 39 participants (59.1\%) with halitosis had asked their dentist for help.

Endoscopic reflux classifications

All patients were grouped according to the LA GERD classification. Sixteen participants were classified as grade A or B esophagitis (24.2\%), and grade $\mathrm{C}$ was diagnosed in 16 patients $(24.2 \%)$. Only three study subjects had grade D esophagitis (4.5\%). The others showed no signs of reflux ( $47 \%, 31$ subjects), resulting in 35 participants classified as ERD and 31 as NERD.

Halitosis scores

The organoleptic assessment of halitosis according to Rosenberg [18] showed, that most of the participants (66.6 $\%, 44$ subjects) had no or only mild halitosis (grade 0 or 1 ) (Table 1). In the organoleptic assessment of halitosis according to Seemann [19], 12 study subjects $(18.2 \%)$ were rated as grade 0 , and $41(62.1 \%)$ as grade 1 . Only 13 participants $(19.7 \%)$ showed a rating higher than grade 1 (Table 1). The halitosis measurements (VSC) and the organoleptic scores according to the indices of Rosenberg [18] and Seemann 
Table 1 Results of the volatile sulfur compound (VSC) measurements and organoleptic assessment of halitosis in 66 patients with symptomatic GERD expressed as a percentage and compared to historic data from healthy participants from the population of the city of Bern ( $n=418)$ [3]

\begin{tabular}{|c|c|c|c|c|c|c|c|c|}
\hline \multicolumn{3}{|c|}{ VSC measurements } & \multicolumn{3}{|c|}{ Organoleptic assessment, Rosenberg et al. [18] } & \multicolumn{3}{|c|}{ Organoleptic assessment, Seemann [19] } \\
\hline \multirow{2}{*}{$\frac{p p b}{<75}$} & \multicolumn{2}{|c|}{ Subjects $(\%)$} & \multirow{2}{*}{$\begin{array}{r}\text { Grade } \\
0\end{array}$} & \multicolumn{2}{|c|}{ Subjects $(\%)$} & \multirow{2}{*}{$\begin{array}{r}\text { Grade } \\
0\end{array}$} & \multicolumn{2}{|c|}{ Subjects $(\%)$} \\
\hline & $\underline{48.5}$ & $72.1^{\mathrm{a}}$ & & $\underline{15.2}$ & $33.2^{\mathrm{a}}$ & & $\underline{18.2}$ & $33.2^{\mathrm{a}}$ \\
\hline 75 to $<110$ & $\overline{36.4}$ & $23.6^{\mathrm{a}}$ & 1 & $\overline{51.5}$ & $35.3^{\mathrm{a}}$ & 1 & 62.1 & $54.2^{\mathrm{a}}$ \\
\hline 110 to $<150$ & $\underline{9}$ & $3.1^{\mathrm{a}}$ & 2 & $\underline{19.7}$ & $20^{\mathrm{a}}$ & 2 & $\underline{15.2}$ & $10.7^{\mathrm{a}}$ \\
\hline \multirow[t]{3}{*}{$\geq 150$} & $\underline{6}$ & $1.2^{\mathrm{a}}$ & 3 & 12.1 & $9.3^{\mathrm{a}}$ & 3 & 4.5 & $1.9^{\mathrm{a}}$ \\
\hline & & & 4 & $\underline{1.5}$ & $2.2^{\mathrm{a}}$ & & & \\
\hline & & & 5 & $\underline{0}$ & $0^{\mathrm{a}}$ & & & \\
\hline
\end{tabular}

Bold and underlined values indicate data from the current study

${ }^{a}$ data from Bornstein et al. [3]

Table 2 Frequencies (total and relative) of subjective (self-reported) and objective halitosis parameters in NERD and NERD patients

\begin{tabular}{|c|c|c|c|c|c|c|c|}
\hline & & \multicolumn{3}{|c|}{$\begin{array}{l}\text { ERD according to LA } \\
\text { classification }\end{array}$} & \multirow{2}{*}{$\begin{array}{l}\text { ERD (total) } \\
n=35\end{array}$} & \multirow{2}{*}{$\begin{array}{l}\text { NERD } \\
n=31\end{array}$} & \multirow[t]{2}{*}{$\begin{array}{l}\text { Correlation, ERD/NERD } \\
\text { (Kendall's tau) }\end{array}$} \\
\hline & & $\begin{array}{l}\mathrm{A} / \mathrm{B} \\
n=16\end{array}$ & $\begin{array}{l}\mathrm{C} \\
n=16\end{array}$ & $\begin{array}{l}\mathrm{D} \\
n=3\end{array}$ & & & \\
\hline \multirow[t]{5}{*}{ Self-perceived tongue coating } & Don't know & 1 & 4 & 0 & $5(14.3 \%)$ & $7(22.6 \%)$ & \multirow[t]{5}{*}{$0.033(\mathrm{NS})$} \\
\hline & Never & 0 & 2 & 2 & $4(11.4 \%)$ & $7(22.6 \%)$ & \\
\hline & Rarely & 4 & 4 & 0 & $8(22.9 \%)$ & $5(16.1 \%)$ & \\
\hline & Somethimes & 8 & 5 & 0 & $13(37.1 \%)$ & $7(22.6 \%)$ & \\
\hline & Often & 3 & 1 & 1 & $5(14.3 \%)$ & $5(16.1 \%)$ & \\
\hline \multirow[t]{4}{*}{ Self-perceived bad taste } & Never & 3 & 4 & 1 & $8(22.9 \%)$ & $6(19.4 \%)$ & \multirow[t]{4}{*}{$0.013(\mathrm{NS})$} \\
\hline & Rarely & 1 & 5 & 0 & $6(17.1 \%)$ & $6(19.4 \%)$ & \\
\hline & Somethimes & 7 & 2 & 2 & $11(31.4 \%)$ & $11(35.5 \%)$ & \\
\hline & Often & 5 & 5 & 0 & $10(28.6 \%)$ & $8(25.9 \%)$ & \\
\hline \multirow[t]{4}{*}{ Self-perceived bad breath } & Never & 6 & 6 & 1 & $13(37.2 \%)$ & $14(45.2 \%)$ & \multirow[t]{4}{*}{0.072 (NS) } \\
\hline & Rarely & 3 & 5 & 1 & $9(25.7 \%)$ & $5(16.1 \%)$ & \\
\hline & Somethimes & 2 & 2 & 0 & $4(11.4 \%)$ & $9(29.0 \%)$ & \\
\hline & Often & 5 & 3 & 1 & $9(25.7 \%)$ & $3(9.7 \%)$ & \\
\hline \multirow[t]{4}{*}{ Tongue coating index } & None & 0 & 1 & 1 & $2(5.7 \%)$ & $5(16.1 \%)$ & \multirow[t]{4}{*}{0.129 (NS) } \\
\hline & Light & 5 & 4 & 0 & $9(25.7 \%)$ & $11(35.5 \%)$ & \\
\hline & Moderate & 7 & 5 & 1 & $13(37.2 \%)$ & $8(25.8 \%)$ & \\
\hline & Severe & 4 & 6 & 1 & $11(31.4 \%)$ & $7(22.6 \%)$ & \\
\hline \multirow{4}{*}{$\begin{array}{l}\text { Organoleptic assessment, } \\
\text { Seemann [19] }\end{array}$} & None & 4 & 3 & 0 & $7(20.0 \%)$ & $5(16.1 \%)$ & \multirow[t]{4}{*}{0.046 (NS) } \\
\hline & $10 \mathrm{~cm}$ & 10 & 10 & 1 & $21(60.0 \%)$ & $20(64.5 \%)$ & \\
\hline & $30 \mathrm{~cm}$ & 2 & 1 & 2 & $5(14.3 \%)$ & $5(16.1 \%)$ & \\
\hline & $1 \mathrm{~m}$ & 0 & 2 & 0 & $2(5.7 \%)$ & $1(3.3 \%)$ & \\
\hline \multirow{5}{*}{$\begin{array}{l}\text { Organoleptic assessment, } \\
\text { Rosenberg et al. [18] }\end{array}$} & None & 3 & 2 & 0 & $5(14.3 \%)$ & $5(16.1 \%)$ & \multirow[t]{5}{*}{$-0.021(\mathrm{NS})$} \\
\hline & Barely noticeable & 8 & 7 & 2 & $17(48.6 \%)$ & $17(54.8 \%)$ & \\
\hline & Slight & 5 & 4 & 0 & $9(25.7 \%)$ & $4(12.8 \%)$ & \\
\hline & Moderate & 0 & 2 & 1 & $3(8.6 \%)$ & $5(16.1 \%)$ & \\
\hline & Strong & 0 & 1 & 0 & $1(2.8 \%)$ & $0(0.0 \%)$ & \\
\hline VSC measurements & Mean \pm SD & $63(32)$ & $87(50)$ & $96(45)$ & $77(43)$ & $83(55)$ & $-0.010(\mathrm{NS})$ \\
\hline
\end{tabular}

$N E R D$ nonerosive gastroesophageal reflux disease, $E R D$ erosive gastroesophageal reflux disease, $V S C$ volatile sulfur compounds, $N S$ no significant differences 
[19]) correlated significantly, but there was no significant correlation between self-reported halitosis and the halitosis measurements (Table 3).

Findings from the questionnaire regarding self-perceived tongue coating, bad taste, and bad breath are comparable in both NERD and ERD patients (Table 2). No statistically significant correlations could be detected between selfperceived halitosis and both organoleptic grading systems $(p<0.777$ and $p<0.779)$ and the VSC values $(p<0.242)$.

The mean VSC values in ERD and NERD patients were $77 \mathrm{ppb}( \pm$ SD 43$)$ and $83 \mathrm{ppb}( \pm$ SD 55$)$, respectively (Table 2$)$. No statistically significant difference could be found between ERD and NERD patients regarding both organoleptic scores and the VSC values (Table 2). No correlation between the severity of the mucosal lesions according to the LA classification and VSC levels as well as organoleptic scores could be detected.

Tongue coating and clinical findings

The tongue was moderately coated (grade 2 ) in $31 \%$ of the patients (21 subjects). In 18 participants $(27.3 \%)$, we found severe coating (grade 3 ), and 20 subjects (30.3\%) showed only a light coating. The remaining subjects $(10.9 \%)$ showed no tongue coating. No statistically significant difference could be found between ERD and NERD patients regarding tongue coating and other anamnestic findings, such as self-perceived bad taste, self-perceived bad breath, and self-perceived tongue coating (Table 2).

\section{Discussion}

In the present study, patients with ERD did not have an increased severity of clinical and/or subjective signs of halitosis compared to patients with NERD. These findings suggest that halitosis is not significantly related to detectable esophageal erosions in conjunction with GERD and seem to support the general observation of interdisciplinary halitosis clinics in several countries that nonoral diseases such as GERD only play a minor role as potential causes for bad breath $[8,21]$.
These data conflict with recent studies [11-15] proposing a relationship between halitosis and GERD. In a crosssectional population-based survey from northern Germany, Struch and coworkers found a positive relationship between self-reported symptoms of GERD and self-reported halitosis [12]. Di Fede et al. observed that patients with endoscopically detectable erosive reflux signs (ERD) exhibited higher levels of selfreported halitosis in comparison to healthy subjects (49.2 versus $31.0 \%$ ) [13]. Another study from Moshkowitz and coworkers revealed a positive correlation between GERD diagnosed by combined endoscopic and clinical examination and self-reported halitosis on a five-point scale [11]. However, it should be noted that self-reported halitosis is not well correlated with objective findings of halitosis, such as organoleptic measurements or instrumental VSC assessments [3, $22,23]$. This lack of correlation is confirmed by data from bad breath clinics $[8,21]$ and by the present study showing no statistically significant correlations between self-reported halitosis and clinical assessments of halitosis by organoleptic measurements and a VSC monitor (Table 3). A recent study from Kinberg and coworkers also demonstrated a correlation between gastrointestinal pathology and halitosis [24], but the authors do not describe their methodology of diagnosing halitosis (self-reported versus organoleptic or VSC measurements). Furthermore, patients taking psychotropic drugs, proton-pump inhibitors, or antibiotics that suppress VSCproducing anaerobic oral microorganisms or patients with systemic illnesses such as diabetes mellitus and liver or renal disease were not excluded from other studies [11-13, 24]. This may affect the results of the halitosis scores and could act as a possible cofounder making it difficult to compare the studies.

For the investigation of a possible relationship between halitosis and GERD clinical data from both ERD and NERD patients, objective halitosis measurements and esophagoduodeno-gastroscopy should be recorded and analyzed. To the best of our knowledge, only two studies from Korea from the same research group documented the relationship between GERD and objective halitosis measurements $[14,15]$. In the first study evaluating a group of 72 patients with self-reported halitosis, higher VSC values were detected in the subgroup

Table 3 Correlation (Kendall's tau coefficients) between the subjective assessment of halitosis in the questionnaire data and halitosis measurements (volatile sulfur compounds (VSC) and organoleptic scores according to the indices of Rosenberg [18] and Seemann [19])

\begin{tabular}{lcccc}
\hline & Organoleptic 1 & Organoleptic 2 & VSC & $\begin{array}{c}\text { Self-perceived } \\
\text { bad breath }\end{array}$ \\
\hline Organoleptic 1 & 1 & - & - & - \\
Organoleptic 2 & $0.744(p<0.0001)$ & 1 & - & - \\
VSC & $0.481(p<0.0001)$ & $0.512(p<0.0001)$ & 1 & - \\
Self-perceived bad breath & $0.030(p=0.777)$ & $0.031(p=0.779)$ & $-0.111(p=0.242)$ & 1 \\
\hline
\end{tabular}

Organoleptic 1 Rosenberg et al. [18], Organoleptic 2 Seemann [19] 
with erosive mucosal damage (ERD, $n=48$ ) compared to the subgroup with no mucosal damage (NERD, $n=24$ ) [14]. The second more recent publication supported these findings using a comparable study design comprising 169 patients [15]. The authors speculated that oral VSC values might be used to distinguish between ERD and NERD and discussed the theory that VSCs responsible for halitosis are generated on the eroded upper gut mucosal surfaces, particularly during early lesion development [14]. However, a correlation between the severity of the mucosal lesions according to the LA classification and the amount of detectable VSC was not demonstrated. In the present study, we could not confirm a significant difference in oral VSC levels or organoleptic halitosis scores between ERD and NERD patients. Furthermore, it was not possible to establish a correlation between the severity of ERD according to the LA classification and the amount of measured VSCs. Thus, our data do not support Kim et al.'s hypothesis [14, 15].

On the other hand, the $\mathrm{pH}$ changes leading to erosive mucosal lesions in the esophagus could affect structures in the oral cavity. This has been shown for erosive tooth wear, which is positively associated with GERD [25]. Besides clearly detectable tooth erosions, slight changes in $\mathrm{pH}$ values theoretically might also influence the oral flora, leading to higher levels of tongue coating followed by an increased production of oral VSCs. This aspect could be a possible explanation for the discrepancy between our data and the data from Korea, as confounding factors from the oral cavity such as tongue coating were not reported, although tongue coating is regarded as the principal source of oral malodor $[8,21]$.

In the present study, a similar hygiene behavior was reported by the study participants as in a previous epidemiologic study performed in the population of the same city and using the identical methods for evaluation of selfreported and clinical data regarding halitosis [3]. In both studies, a high level of dental hygiene awareness was reported by the included subjects. Unfortunately, a comparison between our study and other studies regarding a possible association between gastrointestinal disease and oral malodor is problematic, as the latter did not report the highly relevant hygiene parameters [11,13-15]. Future studies are needed to analyze the correlation between oral hygiene levels and gastroenterological diseases.

To the best of our knowledge, tongue coating, VSC levels, and organoleptic halitosis scores have not been previously compared between ERD and NERD patients. However, in the present study no statistically significant difference for the tongue coating index could be determined between ERD and NERD, which gives no support for the latter theory and leads to the conclusion that erosive mucosal lesions are not associated with increased tongue coating or halitosis. Practical conclusion of the present study is that dentists should ideally be the first healthcare provider to examine patients with complaints of halitosis to exclude possible oral causes. A referral to gastroenterologist should be considered if objective symptoms of oral malodor are present after exclusion of oral causes and if gastroenterological diseases are suspected.

Nevertheless, it has to be taken into account that our study lacks a true control group with healthy subjects. According to Wang et al. and Cho et al., a significant proportion of the population is suffering from subclinical forms of GERD [26, 27], and an esophago-duodeno-gastroscopy in healthy participants arises ethical issues. This was the reason that a true (healthy) control group was not included in the present study.

Within the limitations of this study, it can be concluded that objective measurements of halitosis are not correlated with signs of gastroesophageal reflux diseases. Furthermore, no statistically significant difference could be found between ERD and NERD patients regarding tongue coating index, organoleptic scores, and VSC values as well as selfperceived questionnaire data regarding halitosis.

Acknowledgments The authors thank Stephanie Hayoz, from the Institute of Mathematical Statistics and Actuarial Science, University of Bern, for the statistical analysis. Furthermore, we thank Dr. med. Beat Burckhardt and PD Dr. med. Hans Merki, consultant gastroenterologists, for performing some of the upper gastrointestinal endoscopies.

Conflict of interest The authors declare that they have no conflict of interest.

\section{References}

1. Miyazaki H, Sakao S, Katho Y, Takehara T (1995) Correlation between volatile sulphur compounds and certain oral health measurement in the general population. J Periodontol 66:679-684

2. Lie XN, Shinada K, Chen XC, Zhang GX, Yaegaki K, Kawaguchi $Y$ (2006) Oral malodor-related parameters in the Chinese general population. J Clin Periodontol 33:31-36

3. Bornstein MM, Kislig K, Hoti BB, Seemann R, Lussi A (2009) Prevalence of halitosis in the population of the city of Bern, Switzerland: a study comparing self-reported and clinical data. Eur J Oral Sci 117:261-267

4. Bornstein MM, Stocker LB, Seemann R, Buergin BW, Lussi A (2009) Prevalence of halitosis in young male adults: a study in Swiss army recruits comparing self-reported and clinical data. J Periodontol 80:24-31

5. Van Steenberghe D (1997) Breath malodor. Curr Opin Periodontol 4:137-143

6. Den Broek V, Amwt FL, De Baat C (2007) A review of the current literature on aetiology and measurement methods of halitosis. J Dent 35:327-635

7. Shin K, Yaegaki K, Murata T, Ii H, Tanaka T, Aoyama I, Yamauchi $\mathrm{K}$, Toida T, Iwatsuki K (2011) Effects of a composition containing lactoferrin and lactoperoxidase on oral malodor and salivary bacteria: a randomized, double-blind, crossover, placebo-controlled clinical trial. Clin Oral Invest 15:485-493

8. Quirynen M, Dadamio J, Van Den Velde S, De Smit M, Dekeyser C, Van Tornout M, Vandekerckhove B (2009) Characteristics of 2000 patients who visited a halitosis clinic. J Clin Periodontol 36:970-975

9. Tonzetich J (1977) Production and origin of oral malodor: a review of mechanisms and methods of analysis. J Periodontol 48:13-20 
10. Scully C, El-Maaytah M, Porter SR, Greenman J (1997) Breath odor: etiopathogenesis, assessment and management. Eur J Oral Sci 105:287-293

11. Moshkowitz M, Horowitz N, Leshno M, Halpern Z (2007) Halitosis and gastroesophageal reflux disease: a possible association. Oral Dis 13:581-585

12. Struch F, Schwahn C, WallaschofskI H, Grabe HJ, Voelzke H, Lerch MM, Meisel P, Kocher T (2007) Self-reported halitosis and gastro-esophageal reflux disease in the general population. $\mathrm{J}$ Gen Intern Med 23(3):260-266

13. Di Fede O, Di Liberto C, Occipini G, Vigneri S, Lo Russo L, Fedele S, Lo Muzio L, Campisi G (2008) Oral manifestation in patients with gastro-oesophageal reflux disease: a single-center case-control study. J Oral Pathol Med 37:336-340

14. Yoo SH, Jung HS, Sohn WS, Kim BH, Ku BH, Kim YS, Park AW, Hahm KB (2008) Volatile sulfur compounds as a predictor for esophagogastroduodenal mucosal injury. Gut Liver 2:113118

15. Kim JG, Kim YJ, Yoo SH, Lee SJ, Chung JW, Kim MH, Park DK, Hahm K (2010) Halimeter ppb levels as the predictor of erosive gastroesophageal reflux disease. Gut Liver 4:320-325

16. Nocon M, Kulig M, Leodolter A, Malfertheinder P, Willich SN (2005) Validation of the reflux disease questionnaire for a German population. Eur J Gastroenterol Hepatol 17:229-233

17. Lundell LR, Dent J, Bennett JR, Blum AL, Armstrong D, Galmiche JP, Johnson F, Hongo M, Richter JE, Spechler SJ, Tytgat GNJ, Wallin L (1999) Endoscopic assessment of esophagitis: clinical and functional correlates and further validation of Los Angeles classification. Gut 45:172-180

18. Rosenberg M, Kulkarini GV, Bosy A, Mcculloch CA (1991) Reproducibility and sensitivity of oral malodor measurements with a portable sulphide monitor. J Dent Res 70:1436-1440
19. Seemann R (2006) Measurement of halitosis (in German). In: Filippi A (ed) Halitosis. Patients with oral malodor in daily dental practice (in German). Quintessence, Berlin, pp 39-50

20. Lundgren T, Mobilia A, Hallström H, Egelberg J (2007) Evaluation of tongue coating indices. Oral Dis 13:177-180

21. Seemann R, Bizhang M, Djamchidi C, Kage A, Nachnani S (2006) The proportion of pseudo-halitosis patients in a multidisciplinary breath malodour consultation. Int Dent J 56:77-81

22. Rosenberg M, Kozlovsky A, Gelernter I et al (1995) Self-estimation of oral malodor. J Dent Res 74:1577-1582

23. Miyazaki H, Fujita C, Soh I, Takehara T (1996) Relationship between volatile sulphur compounds and oral conditions in the general Japanese population. In: Van Steenberghe D, Rosenberg M (eds) Bad breath. A multidisciplinary approach. Leuven University Press, Leuven, pp 165-179

24. Kinberg S, Stein M, Zion N, Shaoul R (2010) The gastrointestinal aspects of halitosis. Can J Gastroenterol 24:552-556

25. Wilder-Smith $\mathrm{CH}$, Wilder-Smith P, Kawakami-Wong H, Voronets J, Osann K, Lussi A (2009) Quantification of dental erosions in patients with GERC using optical coherence tomography before and after double-blind, randomized treatment with esomeprazole or placebo. Am J Gastroenterol 104(11):2788-2795

26. Wang PC, Hsu CS, Tseng TC, Hsieh TC, Chen CH, Su WC, Lin HH, Wang CC (2011) Male gender, hiatus hernia and Helicobacter pylori infection associated with asymptomatic erosive esophagitis. J Gastroenterol Hepatol. doi:10.1111/j.1440-1746.2011.06881.x

27. Cho JH, Kim HM, Ko GJ, Woo ML, Moon CM, Kim YJ, Han KJ, Song SY, Cho HG (2011) Old age male sex are associated with increased risk of asymptomatic erosive esophagitis: analysis of data from local health examination by the Korean National Health Insurance Corporation. J Gastroenterol Hepatol 25:1034-1038. doi:10.1111/j.1440-1746.2011.06686.x 